\title{
All-cause Healthcare Costs and Mortality in Patients with Systemic Sclerosis with Lung Involvement
}

\author{
Aryeh Fischer, Amanda M. Kong, Jeffrey J. Swigris, Ashley L. Cole, and Karina Raimundo
}

\begin{abstract}
Objective. Patients with systemic sclerosis (SSc) often develop interstitial lung disease (ILD) and/or pulmonary arterial hypertension (PAH). The effect of ILD and PAH on healthcare costs among patients with SSc is not well described. The objective of this analysis was to describe healthcare costs in patients with newly diagnosed SSc and SSc patients newly diagnosed with ILD and/or PAH in the United States.

Methods. This retrospective cohort analysis was conducted in the Truven Health MarketScan Commercial and Medicare Supplemental healthcare claims databases from 2003 to 2014. Based on International Classification of Diseases-9-Clinical Modification diagnosis codes on medical claims, patients were classified into 3 groups: incident SSc, SSc with incident ILD (SSc-ILD), and SSc with incident PAH (SSc-PAH). Patients were required to have continuous enrollment for 5 years to measure all-cause healthcare costs. Costs (adjusted to US\$) were reported overall and by service type and year following diagnosis. Because of the overlap between groups, statistical comparisons were not conducted.

Results. There were 1957 patients with incident SSc, 219 with incident SSc-ILD, and 108 patients with incident SSc-PAH. Average (mean \pm SD) all-cause healthcare costs over followup were higher for patients with incident SSc-ILD $(\$ 191,107 \pm \$ 322,193)$ or patients with incident SSc-PAH $(\$ 254,425 \pm \$ 240,497)$, compared to patients with incident SSc $(\$ 101,839 \pm \$ 167,155)$. Average annual costs over the 5-year period ranged from $\$ 18,513$ to $\$ 23,268$ for patients with incident SSc, from $\$ 31,285$ to $\$ 55,446$ for patients with incident SSc-ILD, and from $\$ 44,454$ to $\$ 63,320$ for patients with incident SSc-PAH. Costs tended to be the highest in the fifth year of followup.

Conclusion. Among patients with SSc, ILD and PAH can result in substantial increases in healthcare costs. (First Release November 15 2017; J Rheumatol 2018;45:235-41; doi:10.3899/jrheum.170307)
\end{abstract}

Key Indexing Terms:

SYSTEMIC SCLEROSIS

INTERSTITIAL LUNG DISEASE HEALTHCARE COSTS

Scleroderma or systemic sclerosis (SSc) is a rare and chronic autoimmune condition, characterized by varying degrees of vasculopathy and organ fibrosis ${ }^{1}$. Its estimated prevalence is 50 to 300 cases per 1 million people, and estimated incidence is 2.3 to 2.8 cases per 1 million people per year ${ }^{2}$. SSc frequently affects the lungs, manifesting as interstitial lung disease (ILD) and/or pulmonary arterial hypertension (PAH). Depending on the modality used to identify its presence, $30-70 \%$ of patients with SSc develop ILD, and about $2-30 \%$ will develop $\mathrm{PAH}^{1,3,4,5}$. Lung involvement induces significant morbidity and is the leading cause of death in SSc. In 1

From the University of Colorado School of Medicine, Denver, Colorado; Truven Health Analytics, an IBM Company, Cambridge, Massachusetts; National Jewish Health, Denver, Colorado; Genentech Inc., South San Francisco, California, USA.

This analysis was funded by Genentech Inc., and F. Hoffmann-La Roche Ltd. A. Fischer, MD, University of Colorado School of Medicine; A.M. Kong, $M P H$, Truven Health Analytics, an IBM Company; J.J. Swigris, DO, MS, National Jewish Health; A.L. Cole, MPH, Truven Health Analytics;

K. Raimundo, MS, Genentech Inc.

Address correspondence to K. Raimundo, Genentech, 1 DNA Way, South San Francisco, California 94080, USA.

E-mail: raimundo.karina@gene.com

Accepted for publication August 11, 2017. study, $55 \%$ of deaths in SSc were attributed directly to the disease; ILD and PAH were the 2 primary causes ${ }^{6}$. A metaanalysis of 40 studies corroborated those findings ${ }^{7}$.

Patients with SSc incur greater healthcare costs than patients without the disease. Estimated incremental direct annual healthcare costs associated with SSc are nearly $\$ 12,000$ (2009 US\$), and lung involvement further increases costs $^{8}$. The objective of our study was to validate and build on those findings by using nationwide claims databases to assess all-cause use and healthcare costs, as well as all-cause mortality in patients with newly diagnosed SSc, SSc with newly diagnosed ILD, or SSc with newly diagnosed PAH.

\section{MATERIALS AND METHODS}

Data sources. We used data from the Truven Health MarketScan Commercial Claims and Encounters and Medicare Supplemental databases from 2003 to 2014. The databases are large, US-based administrative claims databases that include enrollees with insurance through their employers, a health plan, or Medicare supplemental insurance paid for by a current or former employer. The databases include enrollment information, inpatient and outpatient medical claims, and outpatient pharmacy claims, linked by a unique patient identifier. The data were previously collected, statistically deidentified, and compliant with the Health Insurance Portability and Accountability Act; therefore, Institutional Review Board approval was not

Personal non-commercial use only. The Journal of Rheumatology Copyright @ 2018 . All rights reserved. 
required, in accordance with the policies of our institutions. A subset of employees in the MarketScan data have the potential to be linked to the Social Security Administration death index (SSDI) and these patients can be considered a denominator for mortality analyses. If 1 of these patients died, his or her date of death may be available in the SSDI. Cause of death is not available in the SSDI

Patient selection. Three nonmutually exclusive groups of patients were identified: (1) newly diagnosed (incident) SSc; (2) SSc with newly diagnosed ILD (incident SSc-ILD); and (3) SSc with newly diagnosed PAH (incident SSc-PAH).

For inclusion in the SSc cohort, patients were required to have $\geq 2$ nondiagnostic claims on different days with a diagnosis of SSc [International Classification of Diseases-9-Clinical Modification (ICD-9-CM) 710.1] Nondiagnostic claims are those with procedures other than laboratory or radiology tests that may be used to rule out disease. The date of the first diagnosis was defined as the index date. Incident cases included patients with 6 months of continuous enrollment with no SSc diagnoses (a 6-month "clean period") prior to the index date. Patients were excluded if they were diagnosed with a diffuse connective tissue disease other than SSc or secondary Sjögren syndrome (ICD-9-CM 710.0, 710.3-710.9) in the 12 months after the index date.

Enrollees were considered to have incident SSc-ILD if they had $\geq 2$ nondiagnostic claims on different days with a diagnosis of postinflammatory pulmonary fibrosis, idiopathic pulmonary fibrosis, or lung involvement in SSc (ICD-9-CM 515, 516.3, 516.31, 517.2). Again, the first diagnosis was defined as the index date. Patients were required to have $\geq 2$ nondiagnostic claims on different days with a diagnosis of SSc in the 12 months after the index date and no diagnoses of other diffuse connective tissue diseases (not including Sjögren syndrome), or other conditions associated with diffuse parenchymal lung disease [sarcoidosis (ICD-9-CM 135), hypersensitivity pneumonitis (ICD-9-CM 495.x), pneumoconiosis (ICD-9-CM 500), asbestosis (ICD-9-CM 501), silicosis or talcosis (ICD-9-CM 502), berylliosis and other inorganic dusts (ICD-9-CM 503), unspecified pneumoconiosis (ICD-9-CM 505), radiation fibrosis (ICD-9-CM 508.1), or rheumatic lung (ICD-9-CM 714.81)] during that 12-month period. Patients were required to have 6 months of continuous enrollment in the database prior to index date with no ILD diagnoses to establish a group of incident cases. Similar requirements were used to select incident SSc-PAH (ICD-9-CM 416.0); however, patients with diffuse parenchymal lung disease were not excluded.

All-cause healthcare use and costs. All-cause healthcare use and costs (adjusted to 2014 US\$), including insurer-paid and patient-paid amounts, were measured in the 5 years following the index date for patients who were continuously enrolled during that period. Continuous enrollment was required to ensure that all use and costs were gathered. Analyses considered costs annually and over the full 5-year followup period to examine trends over time. The following service types were gathered: inpatient admissions, emergency room visits, outpatient office visits, other outpatient services (such as laboratory and radiology testing, and medication infusions), and outpatient pharmacy claims overall, specifically claims for PAH therapies.

All-cause survival. For the subset of patients who could potentially be linked to the SSDI, time from index date to death was calculated for those who died, and time for index date to censoring (end of continuous enrollment or end of study data) was calculated for those who did not have a death date in the data. No followup requirement was applied to the subset of patients linked to the SSDI for the mortality analysis.

Patient characteristics. Demographic and clinical characteristics were measured for all patients, regardless of length of continuous enrollment following the index date. Demographics were collected from enrollment information and measured on the index date. Clinical characteristics included the presence of comorbid conditions, medication use, and diagnostic procedures in the 12 months after the index date. Comorbid conditions included those with $\geq 1$ nondiagnostic claim using the relevant diagnosis code. Patients were considered to have gastroesophageal reflux disease (GERD) if they had $\geq 1$ nondiagnostic claim or $\geq 2$ claims for medications to treat the disease. Medication use was based on the presence of $\geq 1$ claim with the relevant drug code on an outpatient medical or outpatient pharmacy claim. Diagnostic tests included those with $\geq 1$ medical claim having the relevant procedure code.

Statistical analyses. Descriptive statistics were generated for patient demographics, clinical characteristics, and healthcare use and costs. Kaplan-Meier plots were generated to display time from index date to death or censoring. Statistical comparisons were not conducted between the 3 patient cohorts because they were not mutually exclusive (i.e., 1 patient could belong to more than 1 cohort) in the main analysis. Therefore, no $\mathrm{p}$ values are presented and inferences about comparisons were made based on the absolute numbers, not on statistical significance. A sensitivity analysis was performed, requiring the cohorts to be mutually exclusive for 5-year costs, with comparisons done using t tests and nonparametric tests, and survival outcomes with comparisons done using log-rank tests. For those comparisons, $\mathrm{p}<0.05$ was considered statistically significant. Analyses were conducted with SAS Versions 9.3 and 9.4.

\section{RESULTS}

Patient sample. Based on our definitions, there were 11,752 patients with incident SSc, 1808 with incident SSc-ILD, and 1223 with incident SSc-PAH (Figure 1). Their demographic and clinical characteristics are presented in Table 1. Average age at first claims-based diagnosis of SSc, ILD, or PAH was 54.9 years, 57.0 years, or 59.9 years, respectively. Typical of SSc, the majority of patients (over $80 \%$ ) in any group were female. Of the comorbid conditions evaluated, the most common were GERD (48.4\%-68.8\%) and systemic hypertension (35.0\%-53.6\%). Use of supplemental oxygen therapy was common among incident patients with SSc-ILD (30.1\%) and patients with incident SSc-PAH (43.8\%). Over $80 \%$ of patients with incident SSc-ILD had a pulmonary function test in the 12 months following the index date. Among patients with incident SSc-PAH, 82.5\% had an echocardiogram and $28.5 \%$ had a right heart catheterization within 12 months after the index date.

Among patients with incident SSc, $12.2 \%$ were diagnosed with ILD, and $8.6 \%$ were diagnosed with PAH in the year after Sc diagnosis. Among patients with incident SSc-ILD, $25.2 \%$ were diagnosed with PAH in the year after ILD diagnosis and among patients with incident SSc-PAH, 41.8\% were diagnosed with ILD in the year after PAH diagnosis. Therefore, patients may have contributed to more than 1 cohort, precluding standard statistical testing. Most commonly, the overlap occurred between incident SSc and either incident SSc-ILD or incident SSc-PAH. Among the 1808 patients with incident SSc-ILD, $963(53.3 \%)$ were also included in the incident SSc group. Among the 1223 patients with incident SSc-PAH, 643 (52.6\%) were also in the incident $\mathrm{SSc}$ group.

All-cause healthcare use and costs. There were 1957 incident SSc, 219 incident SSc-ILD, and 108 incident SSc-PAH patients with 5 years of enrollment after index date. All-cause use and costs are presented in Table 2 and Figure 2. Over the 5 -year period, nearly two-thirds of patients with incident SSc-PAH had at least 1 inpatient admission (64\%) compared to $53 \%$ of patients with incident SSc-ILD and $44 \%$ of patients

Personal non-commercial use only. The Journal of Rheumatology Copyright (C) 2018. All rights reserved 


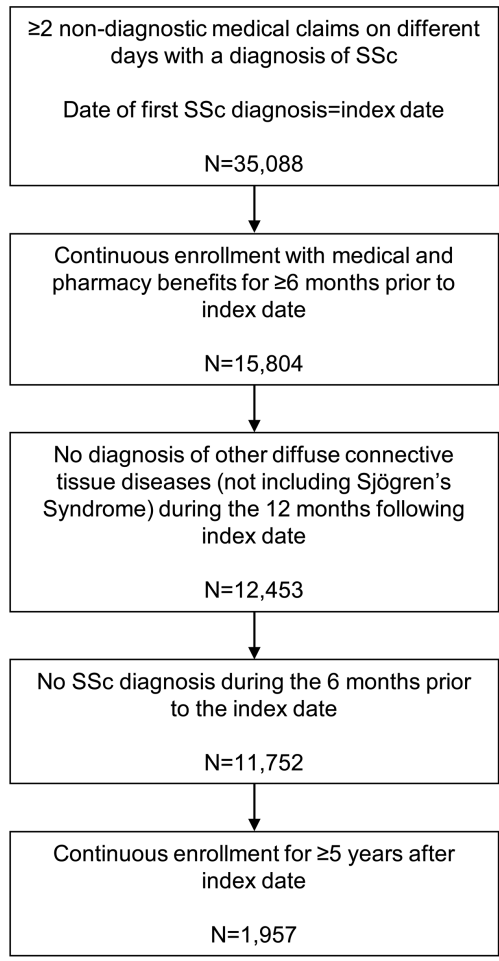

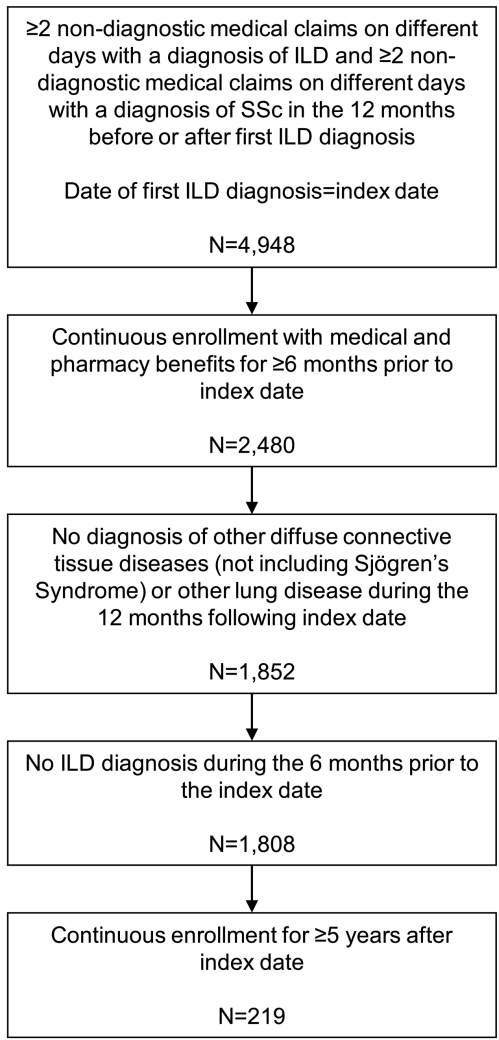

Incident SSc-PAH

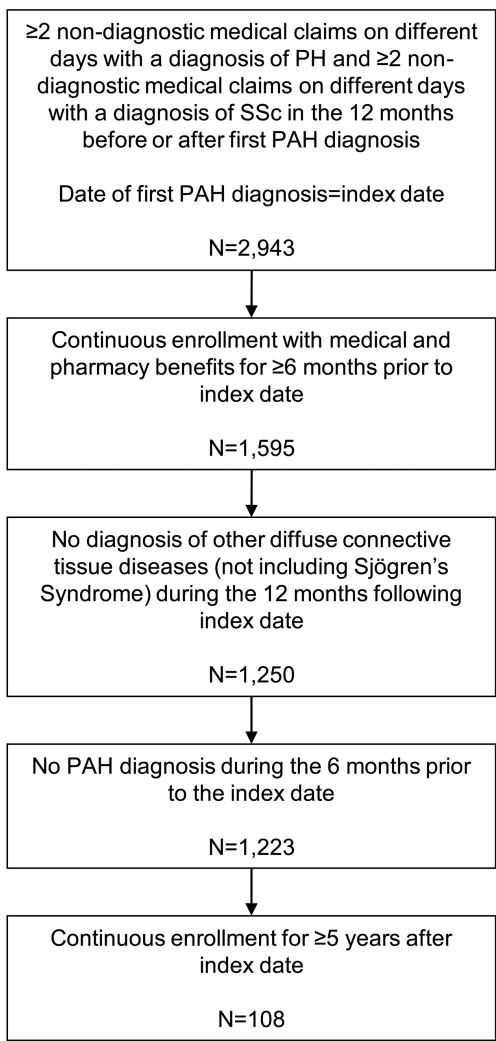

Figure 1. Patient attrition. ILD: interstitial lung disease; PAH: pulmonary arterial hypertension; SSc: systemic sclerosis.

with incident SSc (Table 2). Emergency room visits were also more common among the incident SSc-ILD and incident SSc-PAH groups than among the incident SSc group. Average and median (mean $\pm \mathrm{SD}$; median) all-cause healthcare costs over the full 5-year followup were higher for incident SSc-ILD $(\$ 191,107 \pm \$ 322,193 ; \$ 98,297)$ or incident SSc-PAH $(\$ 254,425 \pm \$ 240,497 ; \$ 171,192)$ compared to incident SSc $(\$ 101,839 \pm \$ 167,155 ; \$ 55,819)$. The majority of costs were for inpatient admissions (16.1\%-25.7\%), other outpatient services (28.2\%-40.0\%), and outpatient pharmacy claims $(30.2 \%-51.3 \%)$. Average costs for all service types were highest for patients with incident SSc-PAH except for inpatient costs. The largest difference was in outpatient pharmacy costs, where incident SSc-PAH patients' costs were more than 4 times that of patients with incident $\mathrm{SSc}$, and nearly double versus patients with incident SSc-ILD. Over the 5-year period, use of PAH therapies was more common among the incident SSc-PAH cohort $(54.6 \%)$ than among the incident SSc $(8.0 \%)$ and incident SSc-ILD cohorts (24.7\%). Average 5-year costs for PAH therapies were $\$ 8519 \pm \$ 44,426$ among patients with incident SSc, $\$ 34,630 \pm \$ 93,161$ for patients with incident SSc-ILD, and $\$ 97,055 \pm \$ 139,289$ for patients with incident SSc-PAH. Similar trends in 5-year costs were seen in mutually exclusive cohorts; however, sample sizes were small for ILD and PAH (Supplementary Table 1, available with the online version of this article).

Average annual costs over the 5-year period ranged from $\$ 18,513$ to $\$ 23,268$ for patients with incident SSc, $\$ 31,285$ to $\$ 55,446$ for patients with incident SSc-ILD, and $\$ 44,454$ to $\$ 63,320$ for patients with incident SSc-PAH (Figure 2 and Supplementary Tables 2-4, available with the online version of this article). Annual costs tended to be highest in the fifth year of followup, compared to earlier years. An increase in average total costs from Year 3 to Year 5 was seen for all 3 groups. For patients with incident SSc-ILD and patients with incident SSc-PAH, the proportion with a hospitalization was highest in years $1(\mathrm{ILD}=21 \%, \mathrm{PAH}=31 \%)$ and 5 (ILD $=25 \%$, PAH $=30 \%$; Supplementary Tables 3-4). Among incident SSc-ILD, the proportion of patients with an emergency room visit tended to increase over time (Supplementary Table 3).

All-cause mortality. There were 4605 patients with incident SSc (39.2\%), 715 patients with incident SSc-ILD (39.5\%), and 525 patients with incident SSc-PAH $(42.9 \%)$ with the potential to be linked to the SSDI. Of those, 579 patients with incident SSc, 159 patients with incident SSc-ILD, and 164 patients with incident SSc-PAH died. Kaplan-Meier curves for survival are presented in Figure 3. Median survival time was 11.3 years, 8.8 years, and 6.0 years in patients with

\section{Personal non-commercial use only. The Journal of Rheumatology Copyright $\subset$ $\odot 2018$. All rights reserved.}


Table 1. Characteristics of patients with incident SSc and patients with incident SSc-ILD or SSc-PAH. Values are n (\%) unless otherwise specified.

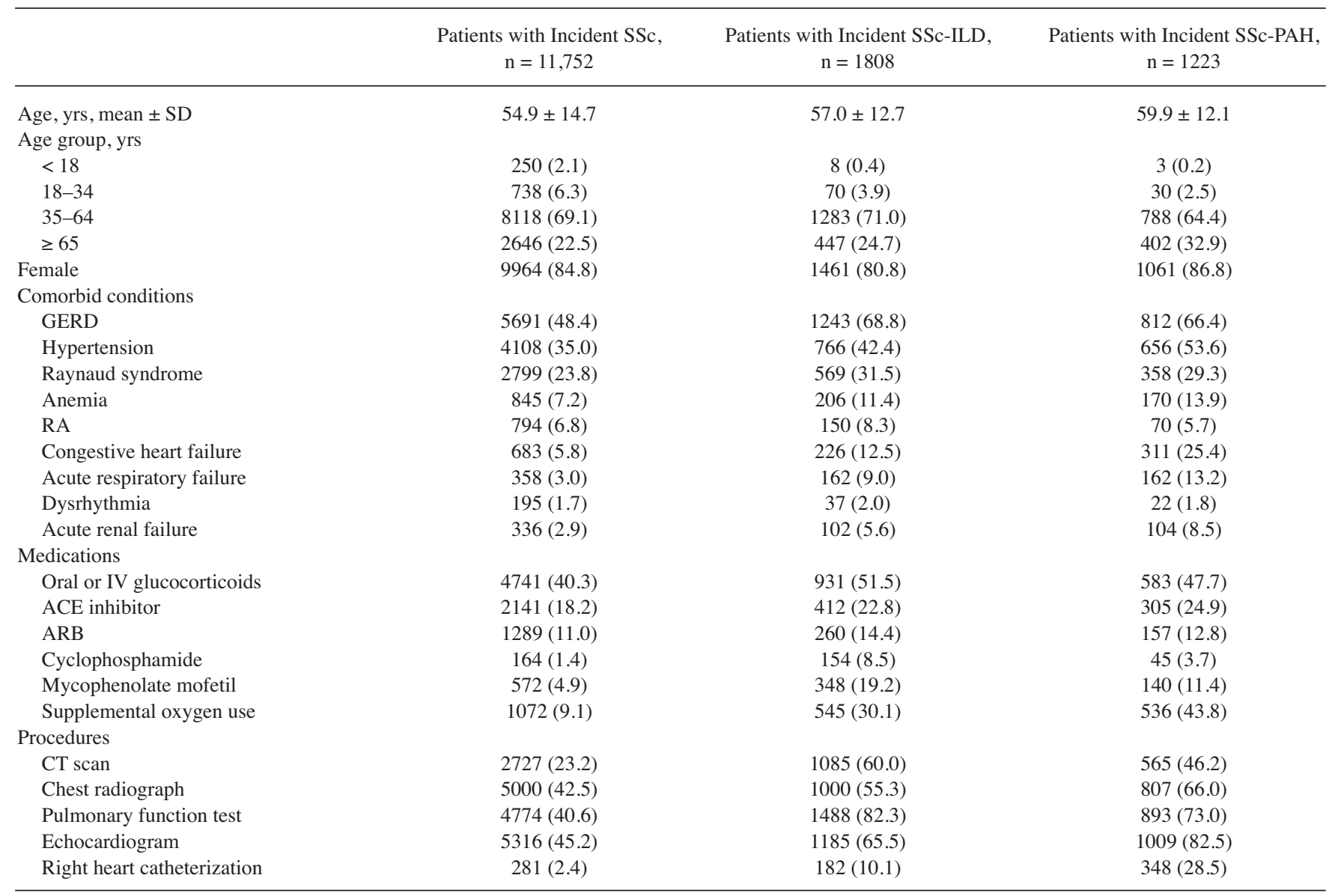

ACE: angiotensin-converting enzyme; ARB: angiotensin II receptor blocker; CT: computed tomography; GERD: gastrointestinal reflux disease; ILD: interstitial lung disease; IV: intravenous; PAH: pulmonary arterial hypertension; RA: rheumatoid arthritis; SSc: systemic sclerosis.

incident SSc, incident SSc-ILD, and incident SSc-PAH, respectively. Similar findings were seen in mutually exclusive cohorts, and all comparisons between survival were statistically significant $(\mathrm{p}<0.01$, Supplementary Figure 1, available with the online version of this article).

\section{DISCUSSION}

We used nationwide claims databases to show healthcare use and costs for patients with SSc. Compared to patients with incident SSc, those with incident SSc-ILD or SSc-PAH had nearly 2 and 2.5 times higher healthcare costs over a 5 -year followup period, respectively. Costs tended to be highest in the fifth year after diagnosis, which may reflect disease progression. PAH may have a larger effect on healthcare costs owing to the availability and costs associated with administering an expanding list of PAH-targeted therapies.

Not surprisingly, published data confirm that patients with SSc incur more healthcare costs than individuals without the disease. A claims-based study of patients with SSc diagnosed from 2003 to 2008 and matched controls with similar demographics and comorbidities reported that average medical costs over 12 months were $\$ 17,365$ for patients with SSc compared to $\$ 5508(\mathrm{p}<0.001)$ for controls ${ }^{8}$. Patients with the severest disease incurred the highest $\operatorname{costs}^{8}$. Controlling for SSc disease status, demographic characteristics, and medication use, patients with lung disease, gastrointestinal bleeding, or renal disease had costs about 2 to 3 times higher than patients without those conditions ${ }^{8}$.

Costs for patients with SSc-related ILD mirror costs for patients with idiopathic ILD or PAH. In 1 study, annual healthcare costs for patients with idiopathic pulmonary fibrosis (IPF) were $\$ 26,378$, about $\$ 12,000$ more than age- and sex-matched controls ${ }^{9}$. In a more recent analysis, all-cause healthcare costs for patients with IPF were a staggering $\$ 59,379^{10}$. Similarly, in a study of patients with a diagnosis of PAH (ICD-9-CM 416.0) from 2002 to 2007 (with no evidence of left heart disease, lung diseases, chronic thromboembolic or miscellaneous pulmonary hypertension, and with evidence of a right heart catheterization or echocardiogram procedure prior to pulmonary hypertension diagnosis), investigators observed that per-month costs were $\$ 2023$ for patients with PAH and \$498 for controls ${ }^{11}$.

Personal non-commercial use only. The Journal of Rheumatology Copyright @ 2018 . All rights reserved. 
Table 2. All-cause healthcare costs and use over 5 years of followup for patients with incident SSc and patients with SSc-ILD or SSc-PAH. Costs were adjusted to 2014 US dollars. Values are \$ unless otherwise specified.

\begin{tabular}{|c|c|c|c|}
\hline & $\begin{array}{l}\text { Patients with Incident SSc, } \\
\qquad \mathrm{n}=1957\end{array}$ & $\begin{array}{l}\text { Patients with Incident SSc-ILD, } \\
\qquad \mathrm{n}=219\end{array}$ & $\begin{array}{l}\text { Patients with Incident SSc-PAH, } \\
\qquad \mathrm{n}=108\end{array}$ \\
\hline Inpatient admissions, $\mathrm{n}(\%)$ & $854(44)$ & $115(53)$ & $69(64)$ \\
\hline $95 \% \mathrm{CI}$ & $18,985-26,683$ & $23,943-74,424$ & $23,626-58,288$ \\
\hline Median (IQR) & $0(0-18,304)$ & $5479(0-31,080)$ & $8668(0-37,523)$ \\
\hline Emergency room visits, $\mathrm{n}(\%)$ & $1195(61)$ & $161(74)$ & $78(72)$ \\
\hline Median (IQR) & $323(0-1353)$ & $746(0-2016)$ & $650(0-2504)$ \\
\hline Outpatient office visits, $\mathrm{n}(\%)$ & $1952(100)$ & $219(100)$ & $108(100)$ \\
\hline Costs, mean $\pm \mathrm{SD}$ & $6050 \pm 7501$ & $7683 \pm 4316$ & $8232 \pm 5260$ \\
\hline $95 \% \mathrm{CI}$ & $5718-6383$ & $7109-8258$ & $7228-9235$ \\
\hline Median (IQR) & $4861(3077-7516)$ & 6942 (4672-9979) & $7417(4475-10,628)$ \\
\hline Other outpatient services & $1957(100)$ & $219(100)$ & $108(100)$ \\
\hline Costs, mean $\pm \mathrm{SD}$ & $30,754 \pm 55,094$ & $68,209 \pm 105,301$ & $130,492 \pm 147,139$ \\
\hline $95 \% \mathrm{CI}$ & $28,311-33,196$ & $54,185-82,233$ & $102,424-158,559$ \\
\hline Median (IQR) & $15,233(6040-31,992)$ & $30,515(12,246-71,256)$ & $60,900(19,916-230,045)$ \\
\hline \multicolumn{4}{|l|}{ Total healthcare } \\
\hline Costs, mean $\pm \mathrm{SD}$ & $101,839 \pm 167,155$ & $191,107 \pm 322,193$ & $254,425 \pm 240,497$ \\
\hline $95 \% \mathrm{CI}$ & $94,429-109,250$ & $148,197-234,017$ & $208,549-300,301$ \\
\hline Median (IQR) & $55,819(29,396-107,486)$ & $98,297(55,562-178,791)$ & $171,192(81,246-396,074)$ \\
\hline
\end{tabular}

ILD: interstitial lung disease; IQR: interquartile range; PAH: pulmonary arterial hypertension; SSc: systemic sclerosis.

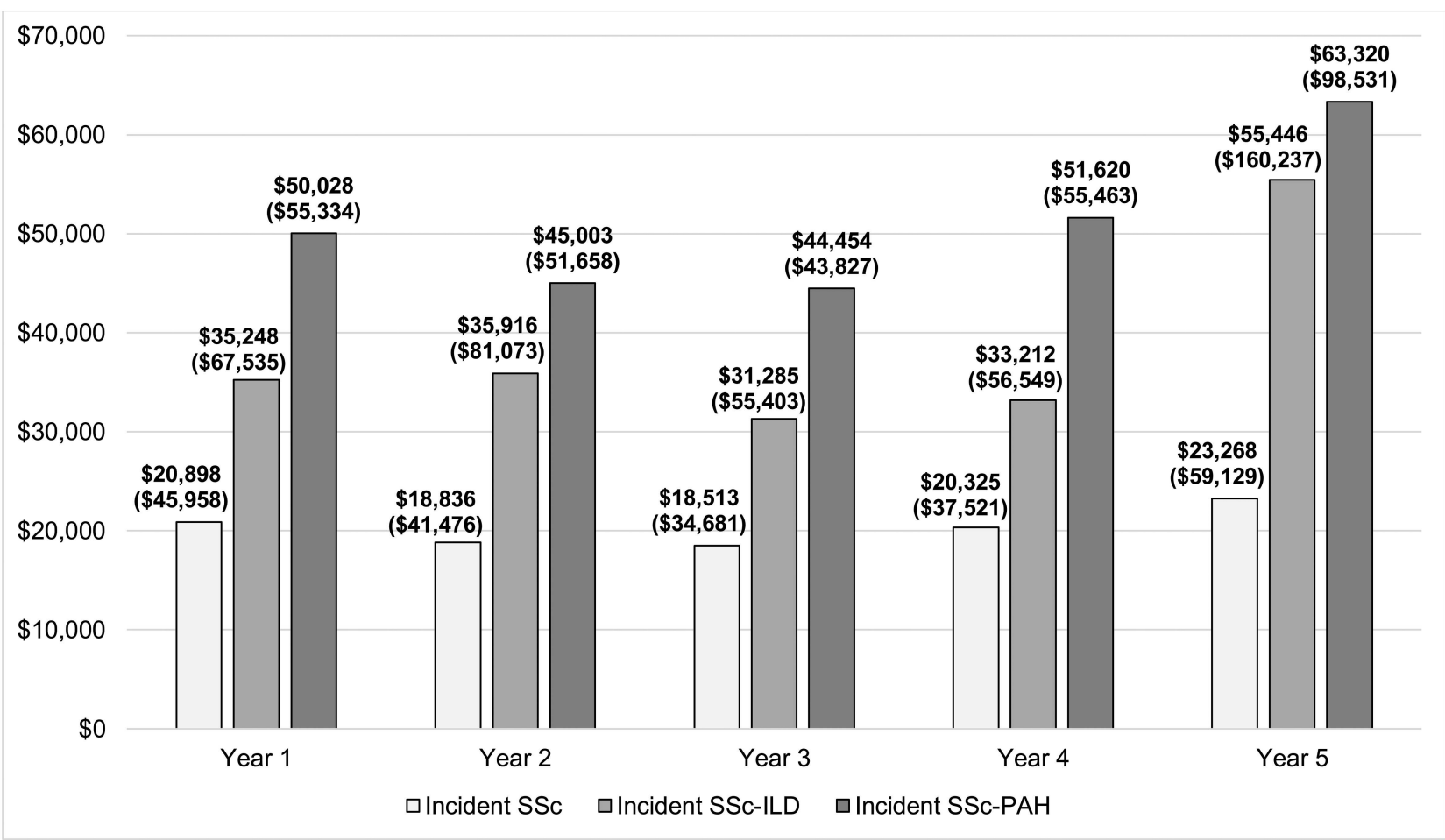

Figure 2. Mean all-cause healthcare costs for incident SSc patients $(\mathrm{n}=1957)$, SSc patients with incident ILD $(\mathrm{n}=219)$, and SSc patients with incident pulmonary arterial hypertension $(n=108)$ by year over 5 years of followup. Costs were adjusted to 2014 US dollars. Values are mean (SD). ILD: interstitial lung disease; PAH: pulmonary arterial hypertension; SSc: systemic sclerosis.

Personal non-commercial use only. The Journal of Rheumatology Copyright (c) 2018. All rights reserved. 


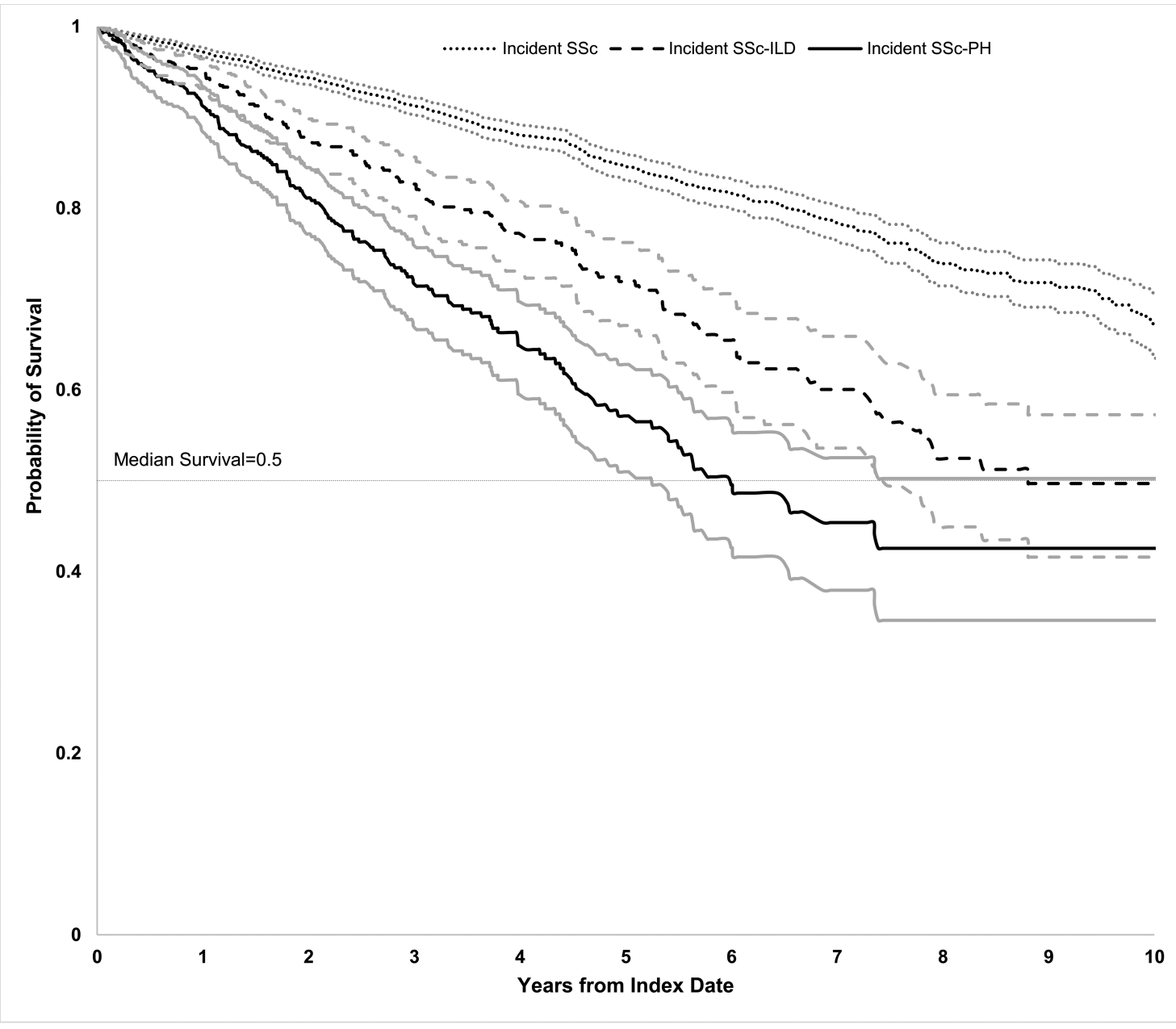

Figure 3. All-cause mortality with 95\% CI for patients with incident SSc $(\mathrm{n}=4605)$, SSc patients with incident ILD $(\mathrm{n}=715)$, and $\mathrm{SSc}$ patients with incident PAH $(\mathrm{n}=525)$. Data were truncated after 10 years. ILD: interstitial lung disease; PAH: pulmonary arterial hypertension; SSc: systemic sclerosis.

Our analysis builds on and extends previously published work by estimating costs in patients with SSc over a period when they develop the 2 deadliest SSc-related manifestations: ILD or PAH. A metaanalysis, including analyses published through July 2013, found that individuals with SSc had a standardized mortality rate (SMR) over 2.5 times higher than the general population $(\mathrm{SMR}=2.72,95 \% \mathrm{CI}$ $1.93-3.83 ; \mathrm{n}=17$ studies), and $74.9 \%$ of patients were alive at 5 years after initial diagnosis ( $\mathrm{n}=43$ studies $)^{7}$. Pulmonary involvement was the most common cause of death $(47.8 \%$ of SSc-related deaths; $\mathrm{n}=40$ studies $)^{7}$. The death rate attributable to pulmonary involvement in patients with SSc increased significantly over time ${ }^{7}$. We found that at 5 years following initial diagnosis, $85 \%$ of patients with SSc were alive. Our higher survival rate may be due in part to our population being in better health because inclusion in this insurance database is linked to employment for most patients.

Our analysis has limitations. First, no comparisons were made between SSc patients with and without ILD, or with and without PAH who were similar in other characteristics. Comparing these patient groups by adjusting for age, duration, severity of SSc disease, and other characteristics that may affect healthcare costs could yield valuable insights. Our sample sizes were too small to conduct stratified analyses. Additionally, our cohorts were not mutually exclusive because there was a limited sample size of patients with only SSc-ILD and SSc-PAH, although we did perform a sensitivity analysis evaluating mutually exclusive cohorts and found trends similar to the main analysis. Comparisons between patients with ILD and PAH identified at the time of SSc diagnosis versus those who develop the conditions later, and comparisons between patients who have 1 condition versus both, may also be worthwhile. Second, claims data are generated for billing purposes, not research, and lack clinical details. We relied on diagnostic coding to identify patients; however, misclassification may have occurred because medical records with information on clinical details and date of first diagnosis were not available. The validity of using

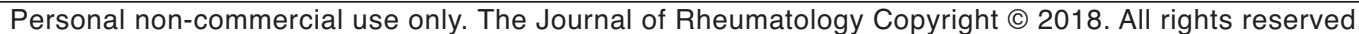


ICD-9-CM 710.1 to identify patients with SSc has been assessed and the use of the diagnosis code was found to be acceptable, with an accuracy rate of $76 \%{ }^{12}$. Little research has been conducted to validate the identification of ILD $^{13}$. One analysis reported that requiring 2 diagnoses of ILD based on diagnosis codes had a positive predictive value of $63 \%$ among patients with autoimmune disease ${ }^{14}$. Although it did not assess validity, another analysis used 2 definitions of ILD among patients with rheumatoid arthritis. One was based on diagnosis codes and receipt of specific tests, while the second definition relied only on the presence of diagnosis codes, as we did in our analysis ${ }^{15}$. Identifying PAH in administrative claims is also challenging. Incident SSc-PAH was based on the diagnostic code of 416.0 only and we cannot reliably determine whether some in the cohort had other forms of pulmonary hypertension and not PAH. Evidence of right heart catheterization in the year following $\mathrm{PAH}$ diagnosis was not seen in all patients and the results of the test are not available in claims data. We were unable to measure prognostically important characteristics of ILD, such as degree of physiologic restriction or extent of ILD by computed tomography scan, or important characteristics of $\mathrm{PAH}$, such as functional class or cardiac hemodynamics measures. Third, the prevalence of comorbid conditions, medication use, and diagnostic procedures may be underestimated because patients were not required to have 12 months of continuous enrollment. Fourth, cost analyses were limited to patients who survived 5 years. This may have resulted in the exclusion of sicker patients who may have died, and the exclusion of end-of-life costs from our calculations. Fifth, information on cause of death is not available in the SSDI data, and mortality may be underestimated given changes to the database available for research ${ }^{16}$. Finally, the study included only individuals with commercial health insurance or Medicare supplemental insurance, and therefore, the results may not be generalizable to other patients. Over $80 \%$ of patients diagnosed with SSc in our study were female, which is consistent with other published studies ${ }^{17,18}$. However, the average age of patients with incident SSc in our study was 55, whereas other analyses have reported mean age at diagnosis to be between 40 and 50 years $^{17,18}$.

In comparison to those with SSc alone, the presence of ILD and/or PAH is associated with substantial increases in healthcare costs and a worse survival experience. Given the effect of lung disease on survival with SSc, these data highlight that there remains an unmet need to identify more effective therapeutic strategies for both ILD and PAH in this patient population. Well-designed studies in the longer term may help further our understanding of the global effect that SSc lung manifestations have on healthcare costs and use.

\section{ONLINE SUPPLEMENT}

Supplementary material accompanies the online version of this article.

\section{REFERENCES}

1. Gabrielli A, Avvedimento EV, Krieg T. Scleroderma. N Engl J Med 2009;360:1989-2003.

2. Chifflot H, Fautrel B, Sordet C, Chatelus E, Sibilia J. Incidence and prevalence of systemic sclerosis: a systemic literature review. Semin Arthritis Rheum 2008;37:223-35.

3. Meyer OC, Fertig N, Lucas M, Somogyi N, Medsger TA Jr. Disease subsets, antinuclear antibody profile, and clinical features in 127 French and 247 US adult patients with systemic sclerosis. J Rheumatol 2007;34:104-9.

4. Hunzelmann N, Genth E, Krieg T, Melchers I, Meurer M, Moinzadeh P, et al. The registry of the German Network for Systemic Scleroderma: frequency of disease subsets and patterns of organ involvement. Rheumatology 2008;47:1185-92.

5. Ferri C, Valentini G, Cozzi F, Sebastiani M, Michelassi C, La Montagna G, et al. Systemic sclerosis: demographic, clinical, and serologic features and survival in 1,012 Italian patients. Medicine 2002;81:139-53.

6. Tyndall AJ, Bannert B, Vonk M, Airò P, Cozzi F, Carreira PE, et al. Causes and risk factors for death in systemic sclerosis: a study from the EULAR Scleroderma Trials and Research (EUSTAR) database. Ann Rheum Dis 2010;69:1809-15.

7. Rubio-Rivas M, Royo C, Simeon CP, Corbella X, Fonollosa V. Mortality and survival in systemic sclerosis: systemic review and meta-analysis. Semin Arthritis Rheum 2014;44:208-19.

8. Furst DE, Fernandes AW, Iorga SR, Greth W, Bancroft T. Annual medical costs and healthcare resource use in patients with systemic sclerosis in an insured population. J Rheumatol 2012;39:2303-9.

9. Collard HR, Ward AJ, Lanes S, Cortney Hayflinger D, Rosenberg DM, Hunsche E. Burden of illness in idiopathic pulmonary fibrosis. J Med Econ 2012;15:829-35.

10. Raimundo K, Chang E, Broder MS, Alexander K, Zazzali J, Swigris JJ. Clinical and economic burden of idiopathic pulmonary fibrosis: a retrospective cohort study. BMC Pulm Med 2016;16:2.

11. Kirson NY, Birnbaum HG, Ivanova JI, Waldman T, Joish V, Williamson T. Excess costs associated with patients with pulmonary arterial hypertension in a US privately insured population. Appl Health Econ Health Policy 2011;9:293-303.

12. Valenzuela A, Yaqub A, Fiorentino D, Krishnan E, Chung L. Validation of the ICD-9-CM code for systemic sclerosis using updated ACR/EULAR classification criteria. Scand J Rheumatol 2015;44:253-5.

13. Jones N, Schneider G, Kachroo S, Rotella P, Avetisyan R, Reynolds MW. A systematic review of validated methods for identifying pulmonary fibrosis and interstitial lung disease using administrative and claims data. Pharmacoepidemiol Drug Saf 2012;21 Suppl $1: 256-60$.

14. Herrinton LJ, Harrold LR, Liu L, Raebel MA, Taharka A, Winthrop $\mathrm{KL}$, et al. Association between anti-TNF- $\alpha$ therapy and interstitial lung disease. Pharmacoepidemiol Drug Saf 2013;22:394-402.

15. Curtis JR, Sarsour K, Napalkov P, Costa LA, Schulman KL. Incidence and complications of interstitial lung disease in users of tocilizumab, rituximab, abatacept and anti-tumor necrosis factor $\alpha$ agents, a retrospective cohort study. Arthritis Res Ther 2015;17:319.

16. da Graca B, Filardo G, Nicewander D. Consequences for healthcare quality and research of the exclusion of records from the Death Master File. Circ Cardiovasc Qual Outcomes 2013;6:124-8.

17. Gelber AC, Manno RL, Shah AA, Woods A, Le EN, Boin F, et al. Race and association with disease manifestations and mortality in scleroderma: a 20-year experience and the Johns Hopkins Scleroderma Center and a review of the literature. Medicine 2013;92:191-205.

18. Mayes MD, Lacey JV Jr, Beebe-Dimmer J, Gillespie BW, Cooper B, Laing TJ, et al. Prevalence, incidence, survival, and disease characteristics of systemic sclerosis in a large US population. Arthritis Rheum 2003;48:2246-55.

Personal non-commercial use only. The Journal of Rheumatology Copyright $\odot$ 2018. All rights reserved. 